\title{
De l'aménagement intégré à la gestion globale des rivières : Les contrats de rivière ont 10 ans
}

\author{
Liliane Duport \\ Chargée de mission au Ministère de l'environnement
}

La procédure des contrats de rivière a été instituée en 1981. Un contrat de rivière est un programme de remise en état d'un cours d'eau dans un délai donné, généralement de 5 ans, sur lequel s'engagent les intervenants: usagers de l'eau et du cours d'eau, et les financeurs institutionnels : collectivités locales, conseils généraux, conseils régionaux, agences de bassin. Le ministère de l'Environnement, pour sa part, apporte une aide incitative de $10 \%$ du montant de certaines opérations.

Ce programme de remise en état doit concerner l'ensemble des aspects de la rivière et des fonctions qu'elle remplit. Il comporte des travaux d'assainissement et d'épuration des collectivités et des industries, des travaux de restauration de la rivière respectueux de l'environnement, des actions de mise en valeur et de préservation des milieux aquatiques, des sites et des paysages ainsi que des actions d'information, de sensibilisation ou encore des actions qui concourent à la gestion de la rivière.

Depuis le $1^{\text {er }}$ avril 1983, et au $1^{\text {er }}$ avril 1991, 31 contrats de rivière ont été signés, 32 sont en projet. Le montant total des travaux engagés est d'environ $1800 \mathrm{MF}$. L'aide du secrétariat d'Etat à l'environnement se monte à $94 \mathrm{MF}$, soit $5 \%$ du montant total. Depuis l'application de la circulaire de 1985 , cette aide ne représente plus que $1 \%$ à $3 \%$ du montant des travaux, soit un montant de subvention par contrat, de 1 à $2 \mathrm{MF}$. Cette aide reste pourtant incitative car elle intervient, le plus souvent, comme les subventions régionales, sur des actions moins subventionnées par ailleurs. L'aide des agences de bassin se situe aux alentours de 10 à $30 \%$. Les aides apportées par les conseils généraux varient d'un département à l'autre, mais sont de l'ordre de $25 \%$. Les conseils généraux comme les conseils régionaux ou les agences de bassin apportent parfois une aide supplémentaire aux opérations programmées dans le cadre de ces contrats de rivière.

La taille des rivières concernées dépasse rarement $200 \mathrm{~km}$ pour la rivière principale, elle est en moyenne d'une centaine de $\mathrm{km}$. Les bassins versants font en moyenne $1700 \mathrm{~km}^{2}$ et les populations riveraines concernées comptent une centaine de milliers de personnes.

La formule du contrat commence à être envisagée sur des rivières importantes comme la Vienne ou la Meuse, le Cher ou la Dordogne; l'expérience manque pour permettre de juger de la pertinence de la démarche contrat de rivière sur de tels cours d'eau compte tenu du nombre forcément élevé des partenaires et du montant important des travaux à engager.

Au total, plus de $4000 \mathrm{~km}$ sont concernés par un contrat ou un projet de contrat. Un contrat intéresse de 7 (Salaison) à 100 communes (Sèvre Nantaise, Drôme) mais plus généralement de 20 à 50 communes. Une dizaine de contrats sont maintenant achevés ou en cours d'achèvement. Des contrats sont entrepris localement sans que des financements d'Etat interviennent (Bas-Léon et Trégor dans le Finistère, Argens dans le Var). La réussite de la politique de gestion globale des rivières se mesurera d'ailleurs lorsque cette pratique des contrats locaux sans incitation de l'Etat, sera devenue courante.

\section{From integrated development to global management of rivers River contracts are ten years old}

Designed in 1977 as instruments to develop quality target maps, river contracts have now become for the Department of the Environment, tools to encourage global management of a waterway. Three circulars - the latest is about to be signed - mark this trend. The drainage part of these contracts usually occurs without any major problems - aid from the Basin agencies are important in this part - but the other parts have greater difficulty in being implemented and are not always carried out in a way that respects the "natural architecture of rivers". For several years, the need has been stressed to have a diagnosis of all sorts of problems on the river before any contract is signed, and a body (intercommunal syndicate, association) is required to manage and coordinate, not just the carrying out of developments on the river, but also its regular upkeep. This upkeep must be set down at the same time as the development programme. 
Trente-trois départements sont concernés par au moins un contrat de rivière en cours, une vingtaine d'autres par un projet. C'est dans les départements du Nord ou dans la région Languedoc-Roussillon qu'existe le plus de projets, 10 dans le premier, 2 signés, 8 dans la seconde mais 4 signés.

Pour 11 seulement des contrats en cours, il existe une structure intercommunale susceptible de prendre en charge ou prenant déjà en charge l'entretien ou la gestion même partielle du cours d'eau. Pour les contrats en projet l'existence de telles structures est une condition presque impérative.

Les actions mises en œuvre concernent, en moyenne, pour $75 \%$ l'assainissement et l'épuration - la reconquête de la qualité des eaux restant le poste le plus important dans le coût des contrats de rivière - , pour $14 \%$ les travaux d'entretien, de restauration de la rivière ou de prévention contre les crues, pour $9 \%$ des aménagements liés à la mise en valeur récréative, l'information et la gestion de la rivière. Si l'on considère les 9 premiers contrats conclus en 1983 et 1984 , ces pourcentages sont respectivement de 80,8 et $1,5 \%$ ( $10 \%$ divers) ; pour les dix derniers contrats signés depuis 1989, ces chiffres sont de 70,12 et 12 .

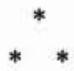

Conçus, à l'origine comme un outil pour la mise en œuvre des cartes d'objectifs de qualité, les contrats de rivière sont devenus des instruments d'incitation vers la gestion des rivières.

Cette évolution est allée de pair avec la prise de conscience et l'émergence d'un certain nombre de faits concernant :

\section{La qualité des eaux}

* La nécessité d'une approche coordonnée de la lutte contre la pollution des eaux et la hiérarchisation des priorités en fonction de l'importance des flux polluants;

* L'«apparition" de nouvelles formes de pollution (la pollution carbonée organique étant traitée mais concentrée en rejets ponctuels) : azote ammoniacal, nitrates et phosphates à l'origine des phénomènes d'eutrophisation, micropolluants. Tous produits obligeant à s'intéresser au bassinversant et à l'occupation des sols ;

* En milieu rural, l'érosion des sols et, en milieu urbain, les eaux pluviales ont des effets de plus en plus importants sur la qualité des eaux notamment sur le taux en matières en suspension et en micropolluants ;

\section{La quantité des eaux}

* La demande croissante d'eau génère un nombre croissant lui aussi de conflits d'usages, mis en évidence ces deux dernières années par la sécheresse. La nécessité de réaliser des schémas d'aménagement des eaux pourtant recommandés depuis la circulaire de 1979, est perçue maintenant à l'évidence ;
* Le changement des régimes hydrauliques consécutifs aux aménagements: en rivière (lutte contre l'érosion des berges, prévention des inondations, barrages) comme sur le territoire du bassin-versant (imperméabilisation des sols) perturbe tout l'équilibre de la rivière ;

\section{Les eaux souterraines}

* La vulnérabilité des nappes aux conséquences de l'intensification économique, agricole ou industrielle, qu'accompagne le développement des villes et des infrastructures mangeuses d'espaces ;

* Les relations nappes-eaux superficielles tant du point de vue de la qualité des eaux que de leur exploitation quantitative et des conflits d'usage qu'elle entraîne;

\section{La protection des milieux}

* La loi sur la pêche de juin 1984 consacre la protection des milieux aquatiques comme intérêt général et oblige à intégrer la préservation de la vie aquatique et la gestion des milieux aquatiques dans la conception des aménagements de la rivière et la gestion de l'eau.

\section{La vie économique}

* La diminution du nombre d'agriculteurs oblige, en milieu rural, d'une part, à la recherche de nouvelles activités parmi lesquelles le tourisme tient une place importante, l'eau constitue alors un atout majeur, et d'autre part, à résoudre les problèmes posés par les terrains en friche (entretien des sols et des berges des cours d'eau...);

* En milieu urbain, les communes tirent de plus en plus partie de la rivière ou du fleuve qui les traverse pour l'amélioration de leur cadre de vie et pour la réhabilitation des quartiers.

\section{L'organisation des institutions}

* La décentralisation, intervenue en 1982, en donnant plus de pouvoirs aux collectivités territoriales a permis que se déconcentre de fait la gestion de l'eau et des rivières. En rapprochant l'objet à gérer du gestionnaire, elle a permis une approche plus systémique, plus globale de l'eau et de la rivière et donc plus politique ;

* Le changement dans la nature des rapports entretenus par les collectivités avec leurs partenaires correspond très bien aux relations contractuelles et au partenariat préconisés dans la politique des contrats de rivière.

L'ensemble des faits énoncés ci-dessus a largement contribué à ce que la gestion des rivières intégrant les aspects sectoriels, techniques ou économiques, aux aspects écologiques et esthétiques voire symboliques, devienne un facteur de la vie politique locale. Les élus alors s'en ressaisissent et en deviennent des animateurs.

Les projets de contrats de rivières débordent maintenant du cadre du seul cours d'eau pour s'intéresser au bassinversant, à la vallée en s'inscrivant dans des projets plus larges de développement local.

Les changements intervenus dans l'approche des problèmes de l'eau depuis dix ans tendent de plus en plus vers 
une démarche de gestion qui se concrétise finalement dans le succès des contrats de rivière même si le nombre de rivières concerné est bien sûr limité. Cette évolution s'est traduite dans les textes ou réflexions qui servent de cadre à la politique des contrats de rivière.

\section{La circulaire du 5 février 1981 :}

Voie contractuelle, recherche du consensus

Anticipant les nouvelles relations partenariales qui font recette maintenant (conséquences des nouveaux rapports introduits par la décentralisation), la circulaire de février 1981 pose le principe de la relation contractuelle, alternative à la voie réglementaire : « le contrat de rivière peut être un instrument de réalisation des cartes d'objectifs de qualité sur certaines rivières en faisant appel, non à la voie réglementaire mais à la voie législative ".

Les objectifs de qualité datent de 1971, la police des rejets est rendue effective en 1973, les incitations financières des agences débutent elles aussi dans les années 1970 ; les opérations exemplaires, à partir d'incitations financières avec les " rivières propres » ou d'actions réglementaires avec les décrets d'objectifs de qualité, n'ont pas donné les résultats escomptés.

La qualité des eaux, meilleure en ce qui concerne la $\mathrm{DB} 05$, se révèle plutôt dégradée en azote, phosphore et bactériologie ; c'est ce que montre l'inventaire de la qualité des eaux de 1981. Un constat mitigé est fait des opérations " rivières propres qui quelquefois traînent en longueur en dépit des aides très incitatives; un seul décret a été signé sur la VIRE et l'expérience n'a pas été jugée concluante. Ce constat fait lors d'une séance du CIQV de mai 1980, est à l'origine de la création des contrats de rivière.

L'idée des contrats de rivière obéit aux principes que pour intéresser les partenaires à la restauration de la qualité des eaux d'une rivière, et donc parvenir à une programmation cohérente et à sa réalisation effective, il fallait que "l'opération ne porte pas seulement sur l'assainissement mais prenne en compte les autres facteurs de la qualité d'une rivière: état du lit, aménagement des berges, etc... " et surtout qu' " un large consensus local se dégage en faveur de l'opération".

Fort de ces principes, le contrat de rivière, convention signée entre l'Etat et le département, après accord écrit de chaque maître d'ouvrage, doit comporter deux volets :

- un volet qualité des eaux ;

- un volet aménagement et protection des berges, du lit de la rivière, du milieu aquatique.

Il s'élabore avec le concours d'" un Comité de rivière " au sein duquel tous les intérêts en cause doivent être représentés.

\section{La circulaire du 12 novembre 1985 :}

Entretien, gestion, information et développement local

Quand paraît la circulaire, 14 contrats sont signés; la formule rencontre donc un certain succès. Un certain nombre de "rivières propres" se sont d'ailleurs reconverties en contrat de rivière, profitant de la dynamique lancée (Sèvre Nantaise ou Armançon demanderont ensuite à bénéficier d'un deuxième contrat). La circulaire de 1985 dresse donc un constat positif. Non seulement les conseils généraux sont signataires mais les régions interviennent aussi et certains contrats font partie des contrats de plan. Cette mobilisation des financeurs institutionnels conduit à penser, en effet, que la rivière est de mieux en mieux perçue, certes encore sur des cas "expérimentaux", comme un atout de développement.

La rivière devient donc un "élément du développement local à protéger et à mettre en valeur ». En conséquence, les projets de contrat devront présenter « un programme global prenant en compte l'ensemble des fonctions du cours d'eau : écoulement, débit et qualité des eaux, milieu naturel et élément du paysage ". Il est, par ailleurs recommandé de veiller «à ce que toutes les conditions soient réunies pour permettre le suivi des actions programmées et assurer la gestion ultérieure et le bon entretien des cours d'eau”.

Trois volets maintenant aux contrats de rivière :

- un volet qualité des eaux ;

- un volet restauration, entretien des berges et du lit, mise en valeur du milieu aquatique et des paysages ;

- un volet concernant des actions d'information ou la gestion du cours d'eau.

\section{Le rapport du groupe de travail sur la gestion intégrée des rivières}

Une communication sur la politique des contrats de rivière présentée en juillet 1986 au CIQV (Comité Interministériel à la Qualité de la Vie) fait le constat du nombre bien sûr limité des rivières concernées par un contrat, elle souligne l'état d'abandon de la plupart des rivières par manque d'entretien régulier et en raison de la façon sectorielle avec laquelle les problèmes sont résolus.

Un groupe de travail interministériel est créé dont la double mission est :

— « d'examiner les dispositions et procédures existantes susceptibles de concourir à la mise en valeur et à la gestion de l'eau et des milieux aquatiques pour prolonger les opérations de réhabilitation résultant des contrats de rivière ". La rédaction d'un guide technique sur la gestion des rivières a été entrepris qui est paru en 1990 ;

— de « rechercher les adaptations à apporter à la politique des contrats de rivière de façon à favoriser la constitution de groupements gestionnaires aptes à déterminer les axes d'une gestion concertée des cours d'eau ».

A l'issue de ses travaux, le groupe de travail conclut à la nécessité d'une gestion globale des cours d'eau, en définit le contenu et souligne l'importance de l'organisation apte à assurer cette gestion. Cette organisation repose de fait sur les collectivités locales et leurs groupements ; elles seules en effet disposent de la légitimité et de moyens pour se substituer aux riverains négligents. Les actions menées par ces structures de gestion doivent néanmoins se situer dans un 
cadre plus large défini par l'administration qui prend en compte les besoins et usages locaux et les besoins et projets extérieurs au territoire, cadre négocié avec tous les partenaires.

\section{L'évaluation des contrats de rivière}

Les premiers contrats arrivant à leur terme une mission d'évaluation est confiée à la mission d'inspection spécialisée en environnement. Les sept rivières visitées ont permis de dégager les aspects positifs des contrats et les problèmes rencontrés.

Parmi les points positifs :

- " la sensibilisation progressive de tous les partenaires potentiels...;

- l'effet mobilisateur du contrat quand le comité de rivière se réunit régulièrement ;

- leur effet médiatique, ...;

- le caractère un peu solennel de la procédure contractuelle qui engage les partenaires et leur fait prendre conscience de leurs solidarités et de leurs responsabilités;

- la participation des régions aux financements;

- le progrès des comportements - réel quoique lent - à l'égard de la valeur patrimoniale des cours d'eau et de la notion de gestion intégrée des cours d'eau, avec la prise en considération de la solidarité des intervenants et de la nécessité d'opérations d'entretien permanent exigeant une organisation collective ;

- l'accélération des opérations d'assainissement industriel par un effet de sensibilisation et de synergie ;

- les résultats acquis sur la qualité des eaux ».

Parmi les points contrariant l'exécution des contrats :

- la faible participation des riverains ;

- les modifications entraînées par le contexte économique ;

- les problèmes de cohérence réglementaire ;

- la cohérence entre les objectifs et certains travaux d'aménagement ;

- l'accès difficile pour l'entretien du cours d'eau;

mais surtout :

- l'absence de structures aptes à assurer la gestion permanente des rivières concernées, l'absence de définition des conditions techniques nécessaires à leur entretien régulier et le coût de cet entretien ;

- le manque d'études générales pour définir les objectifs à poursuivre par l'aménagement de la rivière qui ne sont engagées, dans le meilleur cas, que le contrat signé, différant son exécution ou la remettant en cause.

Tous ces points ont participé à dresser la liste des conditions à inscrire dans la nouvelle circulaire en cours de signature pour l'élaboration d'un contrat de rivière mais plus généralement la liste des conditions nécessaires pour la mise en place du processus de gestion globale des rivières et d'entretien régulier :
— réalisation d'études préalables générales, d'études diagnostic de la rivière ;

- définition d'objectifs à la restauration, l'aménagement et l'entretien de la rivière constituant un véritable "projet pour la rivière " ;

- existence d'une structure (association syndicale, syndicat intercommunal, entente, syndicat mixte, association) susceptible d'assurer d'abord la maîtrise d'ouvrage du contrat puis l'entretien régulier ;

- comité de rivière comprenant une représentation suffisante des entreprises, riverains et usagers dont la présidence serait confiée à une personnalité locale susceptible d'assurer le rôle d'animateur.

Aux trois volets initiaux,

- qualité des eaux,

- restauration des berges et du lit, mise en valeur et protection du milieu aquatique et du paysage,

- entretien et gestion,

il est prévu la possibilité d'adjoindre un volet d'actions ne concernant pas directement la rivière mais pour lesquelles sa réhabilitation constitue une condition de réussite majeure. Ces actions sont directement en rapport avec le développement économique du bassin et concernent, le plus souvent, le développement du tourisme ou l'amélioration du cadre de vie. Elles ne bénéficient pas d'aides particulières du Ministère de l'Environnement mais, faisant partie d'un projet global, d'une stratégie définie au niveau d'un territoire, elles entraînent le plus souvent des financements spécifiques d'autres institutions.

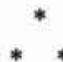

Dans l'évolution qui a conduit d'une gestion intégrée d'une rivière à une gestion globale intégrée à la vie économique, sociale et politique d'un territoire, les contrats de rivière ont constitué et constituent encore un terrain d'expérience tout à fait déterminant même s'ils ne se déroulent pas toujours aussi parfaitement qu'on pourrait l'imaginer ou le souhaiter.

Les nouveaux contrats se dénomment eux-mêmes souvent "contrats de vallée ", c'est le cas pour le Scorff ou la Viosne, par exemple. On constate par ailleurs que des syndicats intercommunaux engagés dans un contrat de rivière sollicitent le bénéfice de l'aide du Ministère de l'Environnement aux plans municipaux d'environnement, engageant par là une réflexion plus globale sur leur environnement. La vallée de la Tech, qui a signé la première convention de ce type, celles de la Drôme, de l'Hérault, de l'Yvette, de l'Orge, la communauté du Béthunois et la vallée de la Lawe, St-Dié et la haute vallée de la Meurthe vont engager cette démarche.

Enfin, le projet de loi sur l'eau en cours de préparation intègre largement ces principes de gestion globale, et il est raisonnable de penser que les contrats de rivière et leurs animateurs ne sont pas totalement étrangers à cette évolution législative. 


\section{Références}

[1] Communication au CIQV de juin 1986 sur le "Bilan des contrats de rivière $"$

[2] Rapport du groupe de travail sur "la gestion globale des rivières " (Secrétariat d'Etat auprès du premier ministre chargé de l'environnement et de la prévention des risques technologiques et naturels majeurs - Conseil général des ponts et chaussées - Mission d'inspection spécialisée en environnement), nov. 1988.

[3] Rapport concernant le bilan des opérations a contrats de rivière " (Conseil général des ponts et chaussées), sept. 1989.

[4] La gestion des rivières (Organisation et environnement et Liliane Duport) - Actions environnement, les cahiers de l'environnement DEPPR, $\mathrm{n}^{\circ} 1$, sept. 1990. 
Theorie des Fleures Elunihe XII.

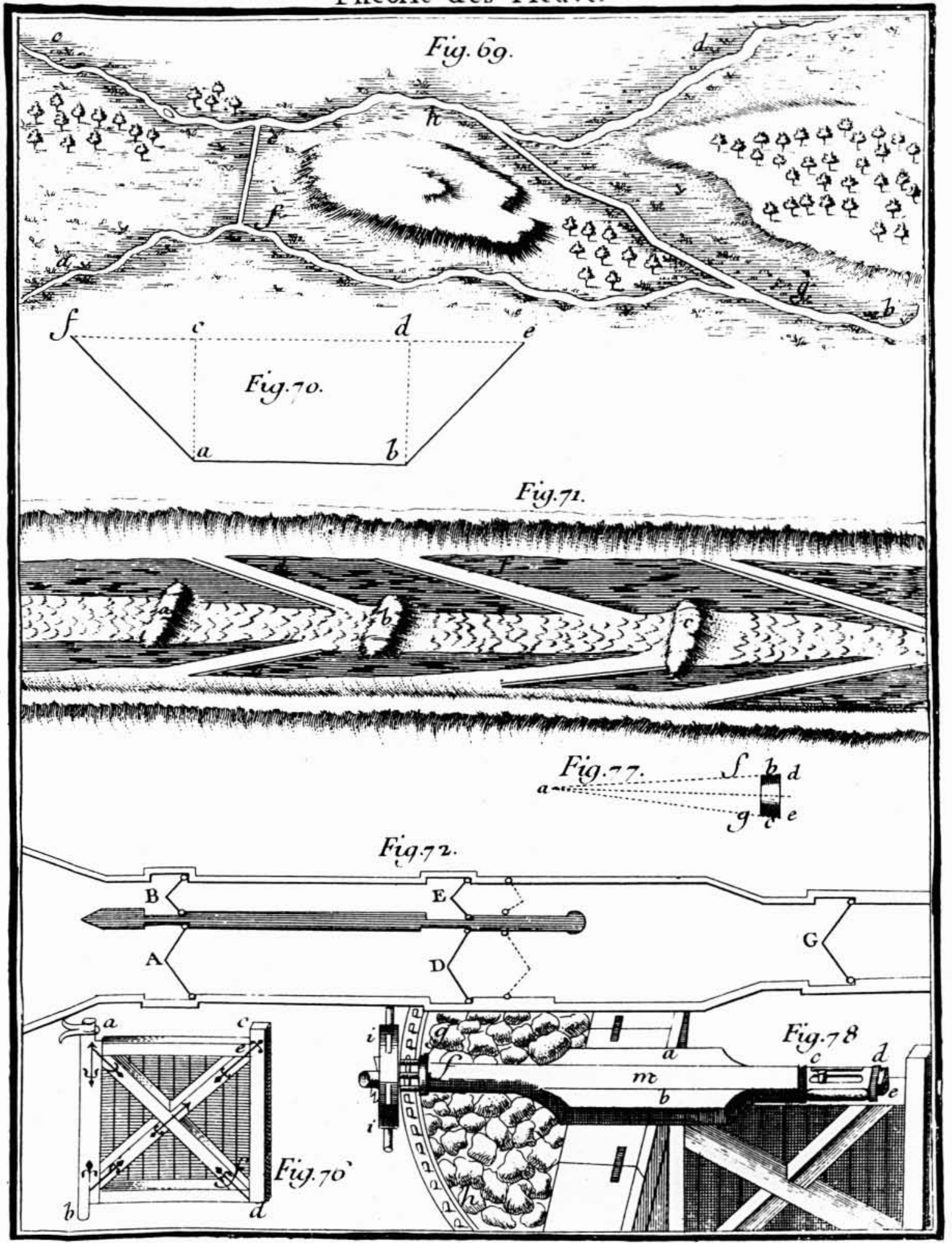

